\title{
Relationship between X(5) models and the interacting boson model
}

\author{
José Barea, ${ }^{1,2, *}$ José M. Arias, ${ }^{2, \dagger}$ and José Enrique García-Ramos ${ }^{3, \ddagger}$ \\ ${ }^{1}$ Instituto de Estructura de la Materia, CSIC, Serrano 123, E-28006 Madrid, Spain \\ ${ }^{2}$ Departamento de Física Atómica, Molecular y Nuclear, Facultad de Física, Universidad de Sevilla, Apartado 1065, E-41080 Sevilla, Spain \\ ${ }^{3}$ Departamento de Física Aplicada, Universidad de Huelva, E-21071 Huelva, Spain
}

(Received 19 April 2010; revised manuscript received 8 July 2010; published 19 August 2010)

\begin{abstract}
The connections between the $\mathrm{X}(5)$ models [the original $\mathrm{X}(5)$ using an infinite square well, $\mathrm{X}(5)-\beta^{8}, \mathrm{X}(5)-\beta^{6}$, $\mathrm{X}(5)-\beta^{4}$, and $\left.\mathrm{X}(5)-\beta^{2}\right]$, based on particular solutions of the geometrical Bohr Hamiltonian with harmonic potential in the $\gamma$ degree of freedom, and the interacting boson model (IBM) are explored. This work is the natural extension of the work presented in García-Ramos and Arias, Phys. Rev. C 77, 054307 (2008) for the E(5) models. For that purpose, a quite general one- and two-body IBM Hamiltonian is used and a numerical fit to the different $\mathrm{X}(5)$ model energies is performed; then the obtained wave functions are used to calculate $B(E 2)$ transition rates. It is shown that within the IBM one can reproduce well the results for energies and $B(E 2)$ transition rates obtained with all these $\mathrm{X}(5)$ models, although the agreement is not so impressive as for the $\mathrm{E}(5)$ models. From the fitted IBM parameters the corresponding energy surface can be extracted and, surprisingly, only the X(5) case corresponds in the moderately large $N$ limit to an energy surface very close to the one expected for a critical point, whereas the rest of models are situated a little further away.
\end{abstract}

DOI: 10.1103/PhysRevC.82.024316

PACS number(s): 21.60.Fw, 21.60.Ev

\section{INTRODUCTION}

In recent years the connection between the Bohr-Mottelson (BM) collective model [1-3] and the interacting boson model (IBM) [4-7] has been the subject of many studies [8-20]. The BM collective model is built on the assumption that the nucleus is composed of a set of strongly interacting fermions that can be treated as a quantum liquid. The surface of such a liquid is characterized in terms of the Hill-Wheeler shape variables $(\beta, \gamma)[21]$ and the Euler angles. Under this approximation, nuclear excitations are small-amplitude vibrations and rotations, corrected by the coupling between them [22]. The IBM was designed to describe the collective quadrupole degrees of freedom in medium-mass and heavy nuclei. The IBM Hamiltonian was written from the beginning in second quantization form in terms of the generators of the $\mathrm{U}(6)$ algebra, subtended by $s$ and $d$ bosons, which carry angular momenta 0 and 2, respectively [7]. Therefore, the connection between the two models is not evident. An approximated connection comes from considering the IBM as the second quantization of the shape variables $(\beta, \gamma)$ [23]. During the 1980s many studies on the connection between the two models were done. The intrinsic state formalism [8-13] was used, but also the complete set of eigenstates [24] was analyzed through a Holstein-Primakoff transformation [25,26] or by an isometric transformation [27]. More recently, the problem of mapping between the two models has been addressed by Rowe and collaborators [14]. Both models present three special limits that can be solved easily and for which the connection between the models is known.

\footnotetext{
*jose.barea@iem.cfmac.csic.es

†ariasc@us.es

†enrique.ramos@dfaie.uhu.es
}

These three cases are (i) the $\mathrm{BM}$ anharmonic vibrator and the dynamical symmetry U(5) IBM limit, (ii) the BM $\gamma$-unstable deformed rotor and the dynamical O(6) IBM limit, and (iii) the $\mathrm{BM}$ axial rotor and the dynamical symmetry $\mathrm{O}(6)$ IBM limit including $Q \cdot Q \cdot Q$ interactions [14,20,28]. Note that, although traditionally accepted, the correspondence of the dynamical symmetry SU(3) IBM limit to a submodel of the BM model has never been explicitly probed [14]. Each of these limits is assigned to a particular shape using the Hill-Wheeler variables $(\beta, \gamma)$ [21]: spherical, deformed with $\gamma$-instability, and axially deformed, respectively. For transitional situations the correspondence between the two models is difficult and a possible way to establish a mapping between the BM model and the IBM is through numerical studies.

Among the transitional Hamiltonians, an especially interesting case occurs when it describes a critical point in the transition from a given shape to another. In general, for such a situation where the structure of the system can change abruptly by applying a small perturbation, both the BM model and the IBM have to be solved numerically. However, a few years ago Iachello proposed schematic Bohr Hamiltonians with the intent of describing different critical points and these can be solved exactly in terms of the zeros of Bessel functions. The first of these models is known as E(5) [29]. E(5) is designed to describe the critical point at the transition from spherical to deformed $\gamma$-unstable shapes. The potential to be used in the differential Bohr equation is assumed to be $\gamma$ independent and, for the $\beta$ degree of freedom, an infinite square well is taken. Similar models were proposed later by Iachello, called $X(5)$ and $Y(5)[30,31]$, to describe the critical points between spherical and axially deformed shapes and between axial and triaxial deformed shapes, respectively. All these models give rise to spectra and electromagnetic transition rates that depend on a couple of free parameters (including a scale). In 
spite of their simplicity, some experimental examples were found $[32,33]$, just after the appearance of these models.

In this work, we concentrate on $\mathrm{X}(5)$ and related models. (The connection between $\mathrm{E}(5)$ and related models and the IBM was already deeply studied in [34].) The formulation of $\mathrm{X}(5)$ attracted immediate attention both experimentally and theoretically. Soon after the introduction of the X(5) model, the nucleus ${ }^{152} \mathrm{Sm}$ was proposed by Casten and Zamfir [33] as a realization of it. Other experimental examples proposed are ${ }^{150} \mathrm{Nd},{ }^{152-154} \mathrm{Gd},{ }^{130} \mathrm{Ce},{ }^{162} \mathrm{Yb},{ }^{166} \mathrm{Hf},{ }^{178} \mathrm{Os},{ }^{226} \mathrm{Ra}$, and ${ }^{226} \mathrm{Th}[35,36]$, although the last two candidates could be better described by combining quadrupole and octupole degrees of freedom [37-39]. Concerning theoretical extensions of X(5), Bonatsos and collaborators studied a sequence of potentials of the type $\beta^{2 n}$, which allows one to go from the vibrational limit, $n=1$, to X(5), $n \rightarrow \infty$ [40]. In particular, in Ref. [40] spectra and transition rates for the potentials of the type $\beta^{2 n}$, with $n \geqslant 1, \beta^{2}, \beta^{4}, \beta^{6}$, and $\beta^{8}$ are given explicitly and compared with the original $\mathrm{X}(5)$ (infinite square well potential) case. Another extension of $\mathrm{X}(5)$ is $\mathrm{X}(3)$, which is a rigid version of X(5) [41]. In Ref. [42] the connection between X(5) and a two-parameter free IBM calculation was studied. In Ref. [43] the authors compared $\mathrm{X}(5)-\beta^{2}, \mathrm{X}(5)-\beta^{4}$, and $\mathrm{X}(3)$ also with a restricted two-parameter IBM calculation with a number of bosons $N=10$. In Refs. [44,45] the authors treated exactly separable versions of the Davidson potential and of the X(5) potential, respectively. Finally, in Ref. [46] the author studied the effect that the $\beta-\gamma$ coupling has in solving the BM equation for the $\mathrm{X}(5)$ potential. As already mentioned, all these models are produced in the BM scheme and a natural question is to ask for the correspondence of them with the IBM. Is the IBM able to produce the same spectra and transition rates? If yes, does the obtained IBM Hamiltonian correspond to a critical point? This work is intended to answer these questions for the $\mathrm{X}(5)$ and related models $\left(-\beta^{8},-\beta^{6},-\beta^{4}\right.$, and $-\beta^{2}$ potentials) and to analyze the convergence as a function of the boson number, $N$.

For that purpose, a large set of $\mathrm{X}(5)$ and related models results for excitation energies and transition rates are taken as reference for numerical fits of the general IBM Hamiltonian. This procedure will allow us to establish the IBM Hamiltonians that best fit the different $\mathrm{X}(5)-\beta^{2 n}$ models and their relation with the critical points.

The paper is organized as follows: In Sec. II the fitting procedure is described and the obtained results are commented upon. Section III is devoted to study of the energy surfaces of the fitted IBM Hamiltonians and to analyzing them in relation to the critical point. Finally, in Sec. IV a summary and conclusions of this work are presented.

\section{THE IBM FIT TO X(5) MODELS}

\section{A. The model}

The most general, including up to two-body terms, IBM Hamiltonian can be written in multipolar form as

$$
\begin{aligned}
\hat{H}= & \varepsilon_{d} \hat{n}_{d}+\kappa_{0} \hat{P}^{\dagger} \hat{P}+\kappa_{1} \hat{L} \cdot \hat{L}+\kappa_{2} \hat{Q} \cdot \hat{Q} \\
& +\kappa_{3} \hat{T}_{3} \cdot \hat{T}_{3}+\kappa_{4} \hat{T}_{4} \cdot \hat{T}_{4},
\end{aligned}
$$

where $\hat{n}_{d}$ is the $d$ boson number operator, and

$$
\begin{aligned}
\hat{P}^{\dagger} & =\frac{1}{2}\left(d^{\dagger} \cdot d^{\dagger}-s^{\dagger} \cdot s^{\dagger}\right), \\
\hat{L} & =\sqrt{10}\left(d^{\dagger} \times \tilde{d}\right)^{(1)}, \\
\hat{Q} & =\left(s^{\dagger} \times \tilde{d}+d^{\dagger} \times \tilde{s}\right)^{(2)}-\frac{\sqrt{7}}{2}\left(d^{\dagger} \times \tilde{d}\right)^{(2)}, \\
\hat{T}_{3} & =\left(d^{\dagger} \times \tilde{d}\right)^{(3)}, \\
\hat{T}_{4} & =\left(d^{\dagger} \times \tilde{d}\right)^{(4)} .
\end{aligned}
$$

The symbol $\cdot$ stands for the scalar product, defined as $\hat{T}_{L} \cdot \hat{T}_{L}=$ $\sum_{M}(-1)^{M} \hat{T}_{L M} \hat{T}_{L-M}$, where $\hat{T}_{L M}$ is the $M$ component of the operator $\hat{T}_{L}$. The operator $\tilde{\gamma}_{\ell m}=(-1)^{m} \gamma_{\ell-m}$ (where $\gamma$ refers to $s$ and $d$ bosons) is introduced to ensure the correct tensorial character under spatial rotations.

The electromagnetic transitions can also be analyzed in the framework of the IBM. In particular, in this work we will focus on the $E 2$ transitions. The most general $E 2$ transition operator including up to one-body terms is written as

$$
\hat{T}_{M}^{E 2}=e_{\mathrm{eff}}\left[\left(s^{\dagger} \times \tilde{d}+d^{\dagger} \times \tilde{s}\right)_{M}^{(2)}+\chi\left(d^{\dagger} \times \tilde{d}\right)_{M}^{(2)}\right],
$$

where $e_{\text {eff }}$ is the boson effective charge and $\chi$ is a structure parameter. In this work $\chi$ will be kept fixed to the $\mathrm{SU}(3)$ value of $-\sqrt{7} / 2$.

Although one could use the most general IBM Hamiltonian (1) to describe the $X(5)$ models a natural question is whether it is possible or not to reduce the number of free parameters. A priori, it is not obvious which terms of the Hamiltonian can be taken out. To answer this question one can rewrite the Hamiltonian (1) in terms of Casimir operators (with the definitions for the Casimir operators having been taken from [47]):

$$
\begin{aligned}
\hat{H}= & \frac{\kappa_{0}}{4} N(N+4)+\left(\varepsilon_{d}+\frac{18}{35} \kappa_{4}\right) \hat{C}_{1}[\mathrm{U}(5)]+\frac{18}{35} \kappa_{4} \hat{C}_{2}[\mathrm{U}(5)] \\
& +\left(\kappa_{1}-\frac{3}{8} \kappa_{2}-\frac{\kappa_{3}}{10}-\frac{\kappa_{4}}{14}\right) \hat{C}_{2}[\mathrm{O}(3)]+\frac{\kappa_{2}}{2} \hat{C}_{2}[\mathrm{SU}(3)] \\
& +\left(\frac{\kappa_{3}}{2}-\frac{3}{14} \kappa_{4}\right) \hat{C}_{2}[\mathrm{O}(5)]-\frac{\kappa_{0}}{4} \hat{C}_{2}[\mathrm{O}(6)]
\end{aligned}
$$

It is expected that for the description of the $\mathrm{X}(5)$ models one will need the contribution of the three IBM dynamical symmetry chains. In the Hamiltonian (8) two contributions come from the $\mathrm{U}(5)$ algebra, the linear and the quadratic Casimir operators. Therefore, it becomes a reasonable "antsatz" to remove the $\mathrm{U}(5)$ quadratic Casimir operator. That implies fixing $\kappa_{4}=0$. Note that its contribution to the rest of the Casimir operators is absorbed by the rest of the parameters.

\section{B. The fitting procedure}

In this section we describe the procedure for getting the IBM Hamiltonian parameters that best fit the different X(5) models. 
The $\chi^{2}$ test is used to perform the fitting. The $\chi^{2}$ function is defined in the standard way,

$$
\chi^{2}=\frac{1}{N_{\text {data }}-N_{\text {par }}} \sum_{i=1}^{N_{\text {data }}} \frac{\left[X_{i}(\text { data })-X_{i}(\mathrm{IBM})\right]^{2}}{\sigma_{i}^{2}},
$$

where $N_{\text {data }}$ is the number of data, from a specific X(5) model, to be fitted, $N_{\text {par }}$ is the number of parameters used in the IBM fit, $X_{i}$ (data) is an energy level [or a $B(E 2)$ value] taken from a particular $\mathrm{X}(5)$ model, $X_{i}(\mathrm{IBM})$ is the corresponding calculated IBM value, and $\sigma_{i}$ is an arbitrary error assigned to each $X_{i}$ (data).

At this point it is necessary to explain how we treat the $\gamma$ bandhead in the $\mathrm{X}(5)$ model. The position of this band is not determined by the model; therefore an extra parameter should be introduced for determining the $\gamma$ bandhead, specifically the location of the bandhead $2_{\gamma}^{+}$state. Consequently, besides the IBM Hamiltonian parameters, there is this extra free parameter, $\Delta_{\gamma}$, in the fit. This parameter gives the optimum position of the $\gamma$ band in a given X(5) model for which the best IBM fit is obtained.

To perform the fit, we minimize the $\chi^{2}$ function for the energies, using $\varepsilon_{d}, \kappa_{0}, \kappa_{1}, \kappa_{2}$, and $\kappa_{3}$ as free parameters of the IBM Hamiltonian and $\Delta_{\gamma}$ as a free parameter for the position of the $\gamma$ bandhead. Note that besides the qualitative argument presented in Sec. II A to justify the election of $\kappa_{4}=0$, we have extensively explored other possibilities, as for example $\kappa_{4} \neq 0$ [48]. For this election a little improvement in $\chi^{2}$ is obtained and for some particular cases it even has a negative impact. In addition, in this case the $\chi^{2}$ function is very flat and the correlation that exists between the free parameters generates nonphysical oscillations in the value of the fitted parameters. We have also explored the case $\kappa_{3}=0$ and $\kappa_{4}=0$, which produces a important increase in the $\chi^{2}$ value. The $\kappa_{4}=0$ selection produces a smooth and consistent behavior of the fitted parameters, as can be observed in Figs. 2-6.

For doing the fit and the minimization of the $\chi^{2}$ function the MINUIT [49] code has been used. It allows us to minimize any multivariable function.

The labels for the energy levels follow the usual notation introduced for the X(5) model [29]: $s$ enumerates the zeros of the $\beta$ part of the wave function, and $n_{\gamma}$ enumerates the number of $\gamma$ phonons. The set of levels included in the fit for the different $\mathrm{X}(5)$ models are as follows:

(i) For the ground-state band, $s=1, n_{\gamma}=0$, the set includes all the states with angular momentum smaller than 10. An arbitrary $\sigma=0.001$ is used for these states except for the $2_{1}^{+}$state for which $\sigma=0.0001$ is used. This latter value allows us to normalize all the IBM energies to $E\left(2_{1}^{+}\right)=1$. Note that the energy of the state $2_{1}^{+}$is fixed arbitrarily to 1 (where we recall that the spectrum is calculated up to a global scale factor).

(ii) For the $\beta$ band, $s=2, n_{\gamma}=0$, the set includes all the states with angular momentum smaller than 10. An arbitrary $\sigma=0.01$ is used for these states.

(iii) For the $s=3, n_{\gamma}=0$ band, which can be identified with the $\beta \beta$ band, the set includes all the states with angular momentum smaller than 10. An arbitrary $\sigma=$ 0.01 is used for these states.
TABLE I. States included in the energy fit. In the states labeled with two subindexes, the first one corresponds to $\mathrm{X}(5)$, whereas the second corresponds to the rest of models.

\begin{tabular}{llc}
\hline \hline Band & Error & States \\
\hline$s=1, n_{\gamma}=0$ & $\sigma=0.0001$ & $2_{1}^{+}$ \\
& $\sigma=0.001$ & $0_{1}^{+}, 4_{1}^{+}, 6_{1}^{+}, 8_{1}^{+}$ \\
$s=2, n_{\gamma}=0$ & $\sigma=0.01$ & $0_{2}^{+}, 2_{2}^{+}, 4_{2}^{+}, 6_{2}^{+}, 8_{2}^{+}$ \\
$s=3, n_{\gamma}=0$ & $\sigma=0.01$ & $0_{3}^{+}, 2_{3,4}^{+}, 4_{4}^{+}, 6_{4}^{+}, 8_{4}^{+}$ \\
$s=1, n_{\gamma}=1$ & $\sigma=0.01$ & $2_{4,3}^{+}, 3_{1}^{+}, 4_{3}^{+}, 5_{1}^{+}, 6_{3}^{+}, 7_{1}^{+}, 8_{3}^{+}$ \\
\hline \hline
\end{tabular}

(iv) For the $\gamma$ band, $s=1, n_{\gamma}=1$, the set includes all the states with angular momentum smaller than 10. An arbitrary $\sigma=0.01$ is used for these states.

With this selection, the number of energy levels included into the fit, $N_{\text {data }}$, is equal to 21 . Note that the state $0_{1}^{+}$is not an actual data point to be reproduced because we are interested just in excitation energies and therefore the ground state is naturally fixed to zero in both the X(5) models and the IBM. In Table I the states included in the fit are explicitly given.

The ordering index for the even $L$ states in the $\gamma, \beta$, and $\beta \beta$ bands is unknown a priori, because of the undetermined position of the $\mathbf{X}(5) \gamma$ bands. However, our best fit always provides a $\gamma$ band above the $\beta$ band, but below the $\beta \beta$ band, that generates the same ordering independently of the particular X(5) model and number of bosons. The only exception happens for $\mathbf{X}(5)$, where the $2^{+}$of the $\gamma$ band is above the $2^{+}$state in the $\beta \beta$ band (i.e., they correspond to $2_{4}^{+}$and $2_{3}^{+}$, respectively).

Once the IBM Hamiltonian is fixed for each $\mathrm{X}(5)$ model by fitting the energy levels, the $\chi^{2}$ function for the $B(E 2)$ values is calculated without any additional fitting. The two parameters in the $E 2$ operator (7) are $e_{\text {eff }}$, which is fixed to give $B\left(E 2 ; 2_{1}^{+} \rightarrow 0_{1}^{+}\right)=100$, and $\chi$, which is fixed to the $\mathrm{SU}(3)$ value of $-\sqrt{7} / 2$. The computed transitions are listed in Table II. Note that only transitions between states with $n_{\gamma}=0$ states are considered.

\section{The results}

We have performed fits of the IBM Hamiltonian (1) parameters plus $\Delta_{\gamma}$ for different values of the number of

TABLE II. $B(E 2)$ transitions to be calculated. $n_{\gamma}=0$ in all the cases.

\begin{tabular}{lccllr}
\hline \hline & $s_{i}$ & $s_{f}$ & & $s_{i}$ & $s_{f}$ \\
\hline$B\left(E 2: 2_{1}^{+} \rightarrow 0_{1}^{+}\right)$ & 1 & 1 & $B\left(E 2: 2_{2}^{+} \rightarrow 0_{1}^{+}\right)$ & 2 & 1 \\
$B\left(E 2: 4_{1}^{+} \rightarrow 2_{1}^{+}\right)$ & 1 & 1 & $B\left(E 2: 2_{2}^{+} \rightarrow 0_{2}^{+}\right)$ & 2 & 2 \\
$B\left(E 2: 6_{1}^{+} \rightarrow 4_{1}^{+}\right)$ & 1 & 1 & $B\left(E 2: 4_{2}^{+} \rightarrow 2_{1}^{+}\right)$ & 2 & 1 \\
$B\left(E 2: 8_{1}^{+} \rightarrow 6_{1}^{+}\right)$ & 1 & 1 & $B\left(E 2: 4_{2}^{+} \rightarrow 4_{1}^{+}\right)$ & 2 & 1 \\
$B\left(E 2: 0_{2}^{+} \rightarrow 2_{1}^{+}\right)$ & 2 & 1 & $B\left(E 2: 4_{2}^{+} \rightarrow 2_{2}^{+}\right)$ & 2 & 2 \\
$B\left(E 2: 2_{2}^{+} \rightarrow 2_{1}^{+}\right)$ & 2 & 1 & & & \\
\hline \hline
\end{tabular}




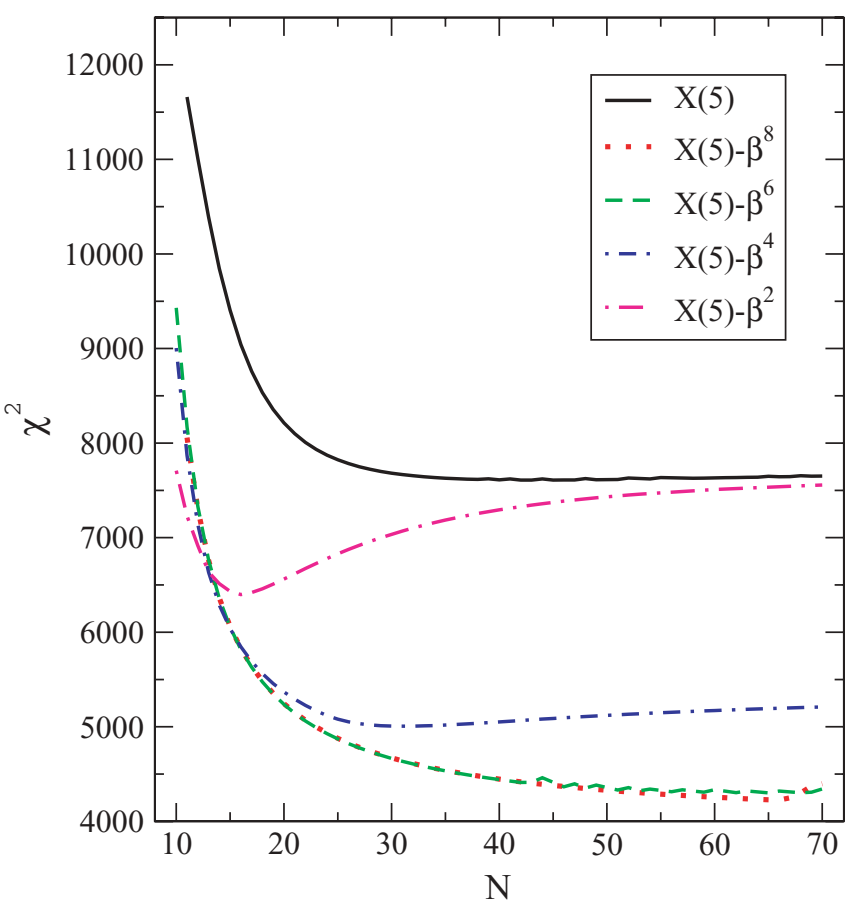

FIG. 1. (Color online) $\chi^{2}$ for the IBM fit to the energy levels of the different $\mathrm{X}(5)$ models, as a function of $N$.

bosons, $N$, so as to reproduce as well as possible the energies of the states given in Table I. These states are generated by the different $\mathrm{X}(5)$ models: $\mathrm{X}(5), \mathrm{X}(5)-\beta^{8}, \mathrm{X}(5)-\beta^{6}, \mathrm{X}(5)-\beta^{4}$, and $\mathrm{X}(5)-\beta^{2}$.

As mentioned before, $\varepsilon_{d}, \kappa_{0}, \kappa_{1}, \kappa_{2}, \kappa_{3}$, and $\Delta_{\gamma}$ are free parameters in a $\chi^{2}$ fit to the energy levels produced by the different $\mathrm{X}(5)$ models (where $\kappa_{4}$ was fixed to zero as discussed in the preceding section). In Fig. 1 the value of the $\chi^{2}$ for the best fit to the different $\mathrm{X}(5)$ models as a function of $N$ is shown. The different lines in Fig. 1 correspond to the fit to different $\mathrm{X}(5)$ models as stated in the legend. It is clearly observed that for any $N$ the best agreement is obtained for the $\mathrm{X}(5)-\beta^{6}$ and $\mathrm{X}(5)-\beta^{8}$ cases, which present a $\chi^{2}$ function almost identical for any value of $N$. The $\chi^{2}$ function increases for $\mathrm{X}(5)-\beta^{4}$ and $\mathrm{X}(5)-\beta^{2}$, up to reach $\mathrm{X}(5)$, which has the higher $\chi^{2}$ value and therefore the worst degree of agreement. Anyway, the differences among the different X(5) models are smaller than a factor 2 in $\chi^{2}$. It is worth noting that these results change slowly with the boson number and in all cases the $\chi^{2}$ function saturates to a given value in the large- $N$ limit. The situation presented here is somehow different from the analysis of the $E(5)$ models [34] where there is a monotonic behavior in the $\chi^{2}$ values in passing from $\mathrm{E}(5)-\beta^{4}$, which presents the lowest value, to $\mathrm{E}(5)-\beta^{6}$, $\mathrm{E}(5)-\beta^{8}$, and $\mathrm{E}(5)$, where the maximum appears.

In Figs. 2-6 the Hamiltonian parameters and the excitation energies for the ground, $\beta$ and $\gamma$ bands are plotted for the cases of $\mathrm{X}(5), \mathrm{X}(5)-\beta^{8}, \mathrm{X}(5)-\beta^{6}, \mathrm{X}(5)-\beta^{4}$, and $\mathrm{X}(5)-\beta^{2}$, respectively. The Hamiltonian parameters present clear analogies in their behavior in all the $\mathrm{X}(5)$ models. $\varepsilon_{d}$ increases continuously on the whole range of $N$ for all the $\mathrm{X}(5)$ models. $\kappa_{1}$, in contrast, exhibits a relatively small and modest value. $\kappa_{0}$ and $\kappa_{2}$ show a very smooth variation until reaching a value of saturation, with a tendency to increase in all cases, except in the case of $\kappa_{0}$ for $\mathrm{X}(5)-\beta^{2}$, which tends to decrease. $\kappa_{3}$ in almost all the cases tends to increase all the way. In the $\mathrm{X}(5)$ case (Fig. 2), $\kappa_{3}$ exhibits a decreasing behavior for small values of $N$ but, as already mentioned, exhibits an increasing behavior for larger $N$ values. A similar behavior is also observed for $\Delta_{\gamma}$. For the
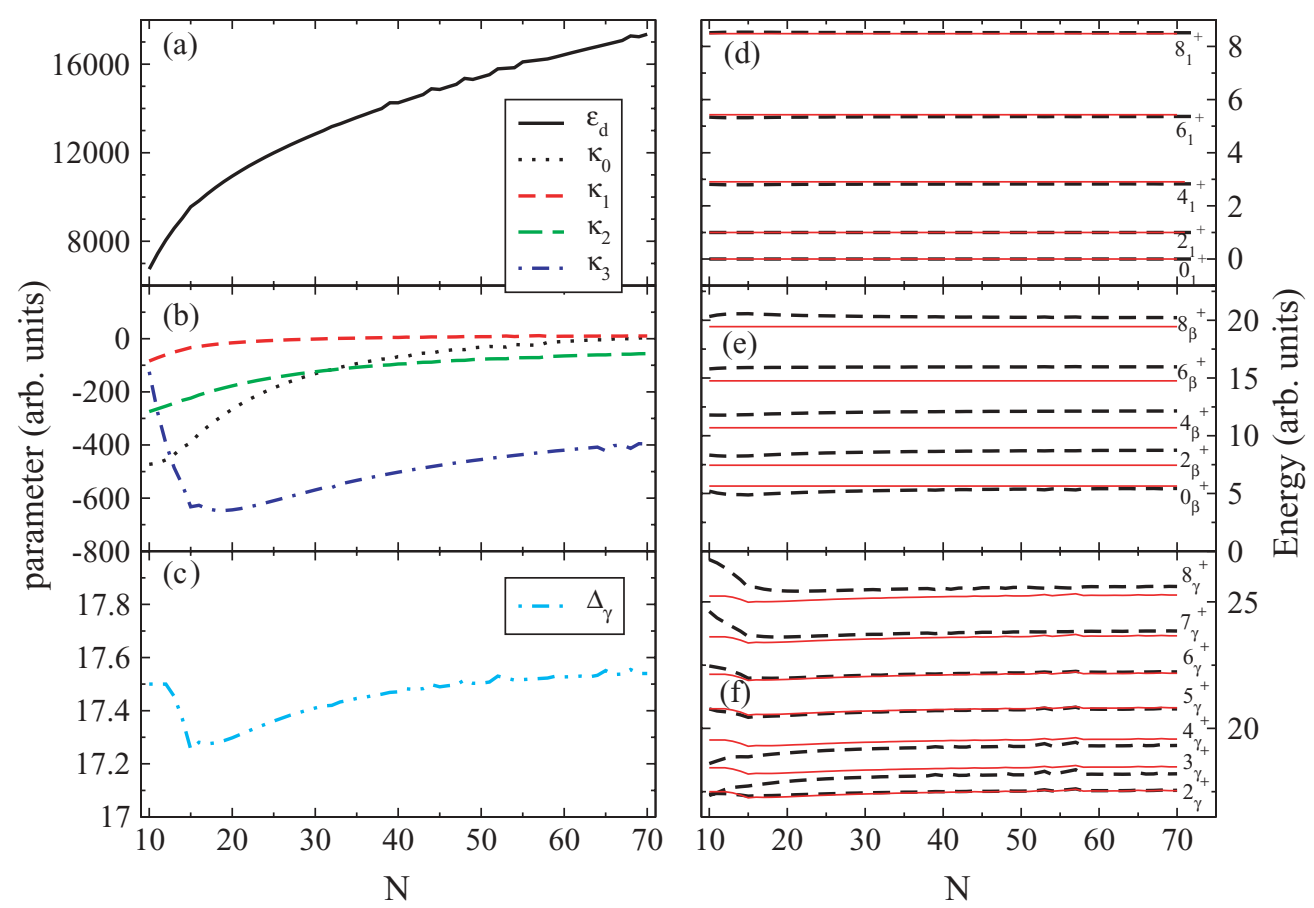

FIG. 2. (Color online) Parameters [(a), (b), and (c)] and excitation energies of the ground (d), $\beta$ (e), and $\gamma$ (f) bands for the X(5) case, as a function of $N$. In the right panels, the continuous red lines are the $\mathrm{X}(5)$ results, and the dashed black lines are the fitted results. 

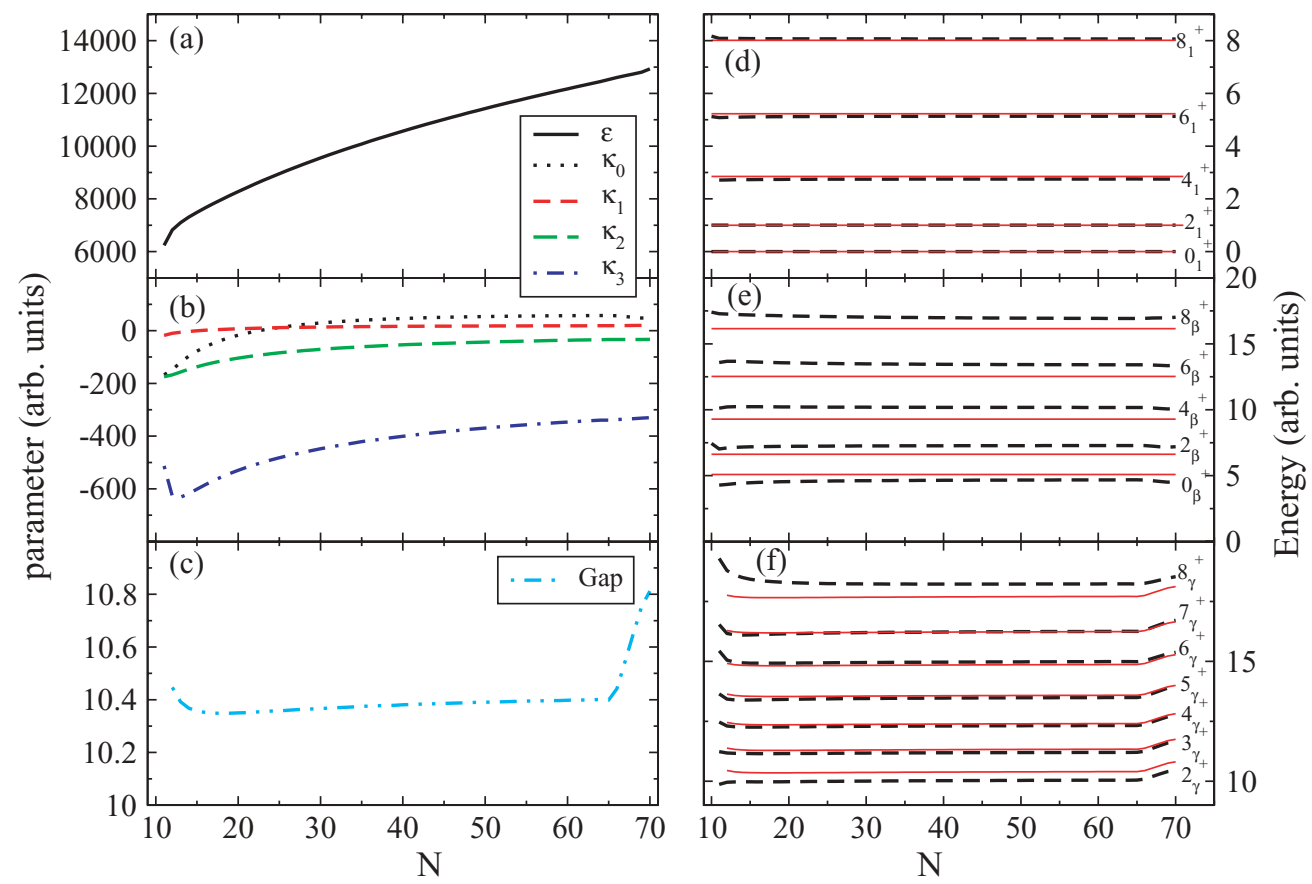

FIG. 3. (Color online) Same as Fig. 2 but for the $\mathrm{X}(5)-\beta^{8}$ case.

energy levels, it is remarkable that the plotted energies have almost constant values regardless the value of $N$, except for the $\gamma$ band in the low- $N$ region where the energies smoothly move to a saturation value. The agreement for the ground-state band is very good but for the $\beta$ and $\gamma$ bands the agreement is poorer. In the $\mathrm{X}(5)-\beta^{8}$ case (Fig. 3), $\kappa_{3}$ also exhibits a decreasing behavior for very low values of $N$, but from there on it monotonically increases. $\Delta_{\gamma}$ exhibits an almost constant value, except for the largest values of $N$, where a increase (note the small energy scale) is shown. The energies remain almost constant in the full range of $N$, except for the $\gamma$ band, where the increase of the $\Delta_{\gamma}$ value generates, for large values on $N$, a corresponding increase. The agreement of the energies in the ground-state band is perfect and reasonable in the $\beta$ and $\gamma$ bands. The $\mathrm{X}(5)-\beta^{6}$ case (Fig. 4 ) is very similar to that of $\mathrm{X}(5)-\beta^{8}$, although here $\Delta_{\gamma}$ exhibits a rather flat behavior with
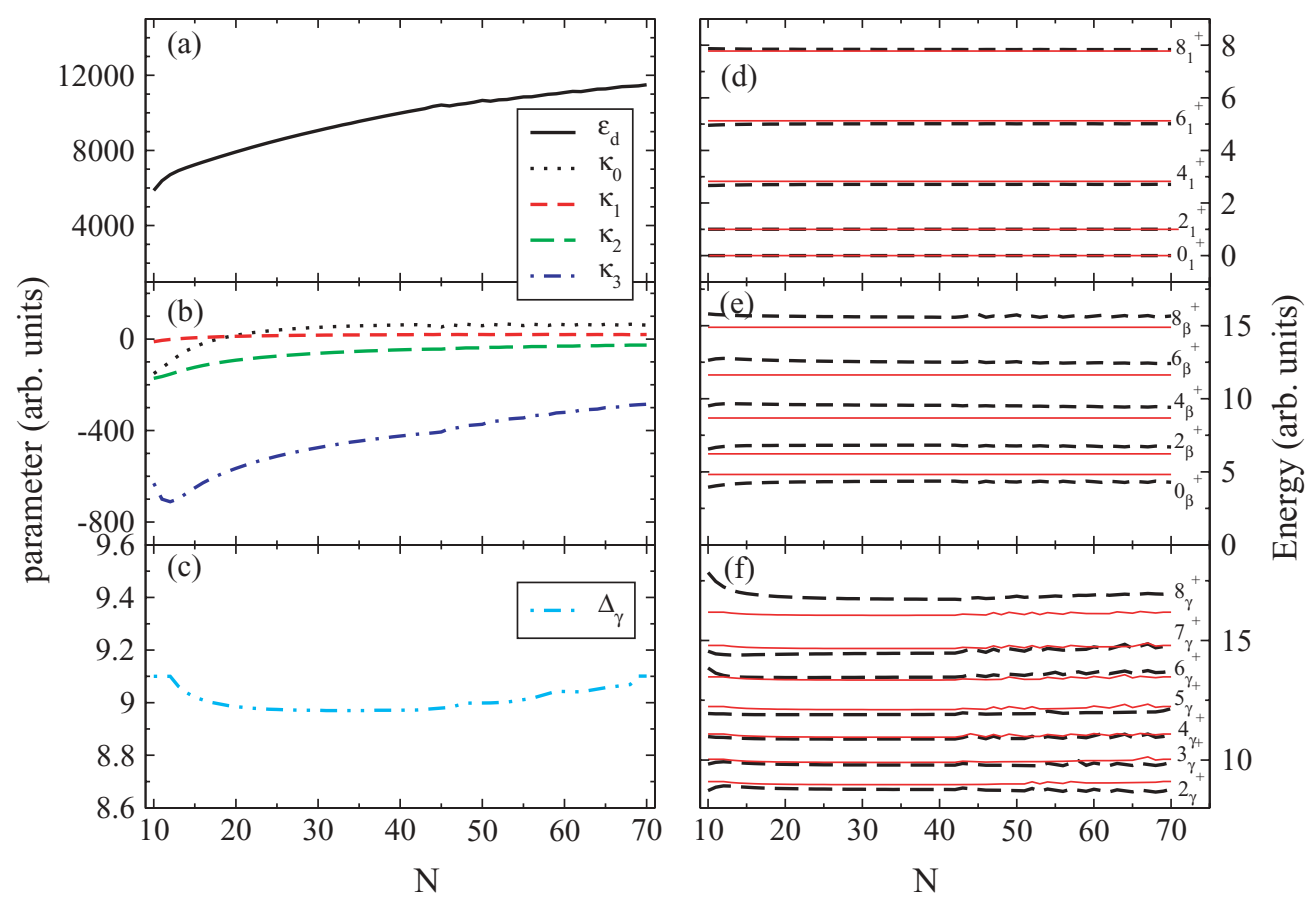

FIG. 4. (Color online) Same as Fig. 2 but for the $\mathrm{X}(5)-\beta^{6}$ case. 

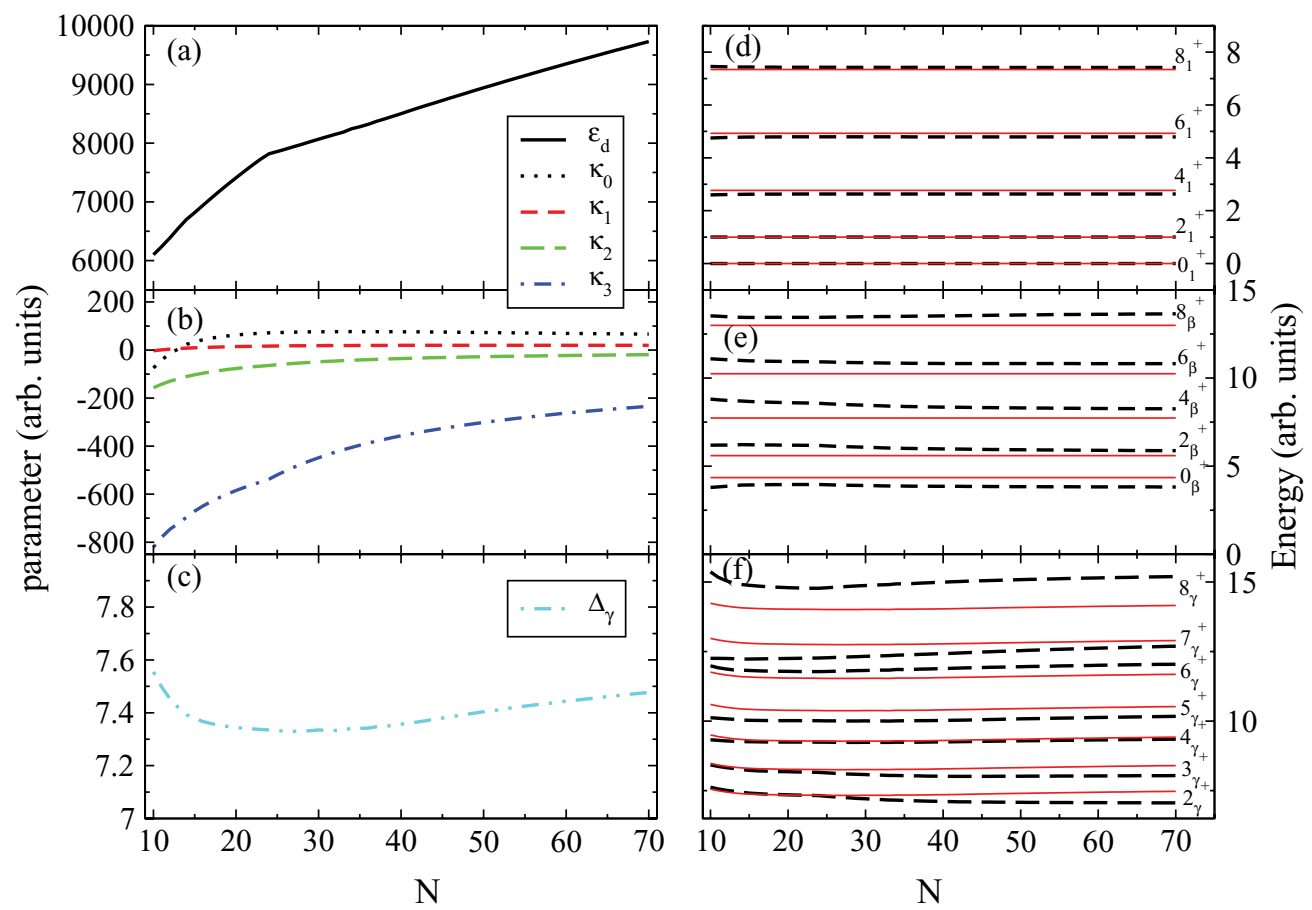

FIG. 5. (Color online) Same as Fig. 2 but for the $\mathrm{X}(5)-\beta^{4}$ case.

a minimum around $N=40$. Once more, the agreement of the energies in the ground-state band is perfect and reasonable in the $\beta$ and $\gamma$ bands. The $\mathrm{X}(5)-\beta^{4}$ case shown in Fig. 5 shows a monotonic increase of $\kappa_{3}$ and a behavior for $\Delta_{\gamma}$ almost identical to that for $\mathrm{X}(5)-\beta^{6}$. Here also the agreement of the energies in the ground-state band is good whereas the description of the $\beta$ and $\gamma$ bands is poorer. Finally, $\mathrm{X}(5)-\beta^{2}$ (Fig. 6) also exhibits a monotonic increase of $\kappa_{3}$ whereas $\kappa_{0}$ has a smooth decreasing behavior till a saturation value. In $\Delta_{\gamma}$ the behavior of $\mathrm{X}(5)$ is recovered, with a smooth decrease up to
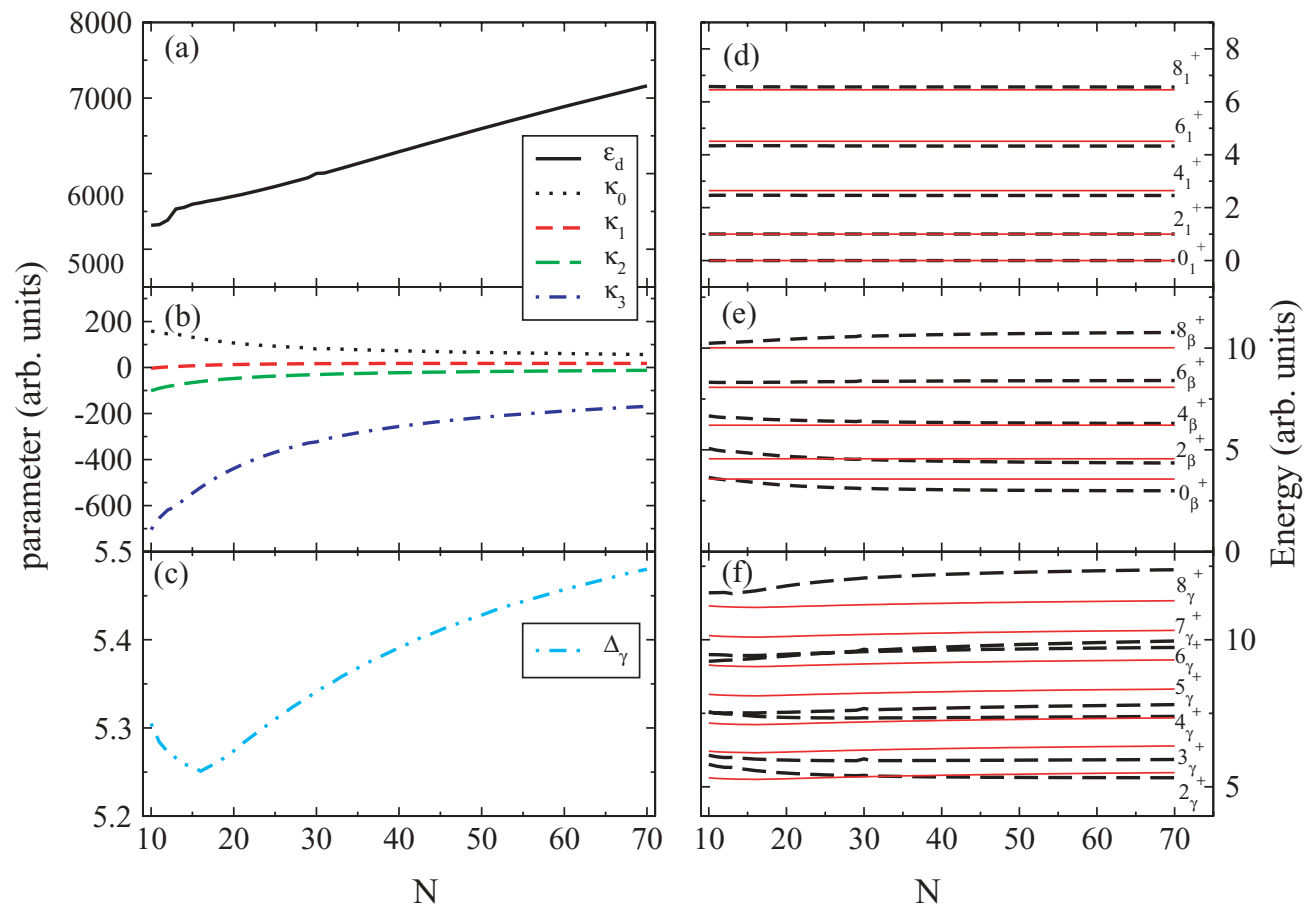

FIG. 6. (Color online) Same as Fig. 2 but for the $\mathrm{X}(5)-\beta^{2}$ case. 
TABLE III. Parameters (in arbitrary units) of the IBM Hamiltonian plus the excitation energy for the $\gamma$ bandheads, $\Delta_{\gamma}$, that best fit the different $\mathrm{X}(5)$ models for $N=50$.

\begin{tabular}{lrrrcrr}
\hline \hline & \multicolumn{1}{c}{$\varepsilon_{d}$} & \multicolumn{1}{c}{$\kappa_{0}$} & \multicolumn{1}{c}{$\kappa_{1}$} & \multicolumn{1}{c}{$\kappa_{2}$} & \multicolumn{1}{c}{$\kappa_{3}$} & \multicolumn{1}{c}{$\Delta_{\gamma}$} \\
\hline $\mathrm{X}(5)$ & 15420 & -31.7 & 7.5 & -77.1 & -454.8 & 17.5 \\
$\mathrm{X}(5)-\beta^{8}$ & 11426 & 53.6 & 17.8 & -43.3 & -369.2 & 10.4 \\
$\mathrm{X}(5)-\beta^{6}$ & 10661 & 58.5 & 19.9 & -38.5 & -372.7 & 9.2 \\
$\mathrm{X}(5)-\beta^{4}$ & 8942 & 72.9 & 19.6 & -27.8 & -301.4 & 7.4 \\
$\mathrm{X}(5)-\beta^{2}$ & 6595 & 65.9 & 18.2 & -17.6 & -216.6 & 5.4 \\
\hline \hline
\end{tabular}

$N=15$ and a smooth increase from there on. The agreement of the energies in the ground-state band is very good and reasonable for the $\beta$ and $\gamma$ bands.

In panels (e) of Figs. 2-6 one can see that the moment of inertia of the $\beta$ band increases from $\mathrm{X}(5)$, where it reaches the smallest value, till $\mathrm{X}(5)-\beta^{2}$, where it is maximum. In panels (d) and (f) of these figures one can also observe how the moment of inertia of ground and $\gamma$ bands is roughly stable for all the models. This tendency is correctly reproduced by the IBM fits.

It is worth remarking that the agreement of the energies in the $\beta$ and the $\gamma$ bands does not clearly deteriorate when ascending up in the band. In particular, in the $\gamma$ band the best agreement is obtained for angular momenta values 4,5 , and 6. Similar conclusions are obtained for the $\beta \beta$ band. This fact has a important consequence for the value of $\chi^{2}$ : Contrary to what might be expected, the higher states included in the fitting procedure for the $\beta, \gamma$, and $\beta \beta$ bands produce smaller contributions to $\chi^{2}$ than the low-lying states of these bands.

To have a clearer idea of the values of the Hamiltonian parameters obtained in the fits we present in Table III the parameters of the IBM Hamiltonians that best fit the different $\mathrm{X}(5)$ models for $N=50$. Two facts are apparent: 1 . The parameters for $\mathrm{X}(5)-\beta^{8}$ and $\mathrm{X}(5)-\beta^{6}$ are amazingly similar and 2 . The parameters change almost monotonically when going from $\mathrm{X}(5)$ to $\mathrm{X}(5)-\beta^{2}$.

As a test for the produced wave functions with the fitted IBM Hamiltonian, they are used for calculating E2 transition probabilities, $B(E 2)$. The effective charge in the $E 2$ operator (7) is fixed so as to give $B\left(E 2 ; 2_{1}^{+} \rightarrow 0_{1}^{+}\right)=100$,

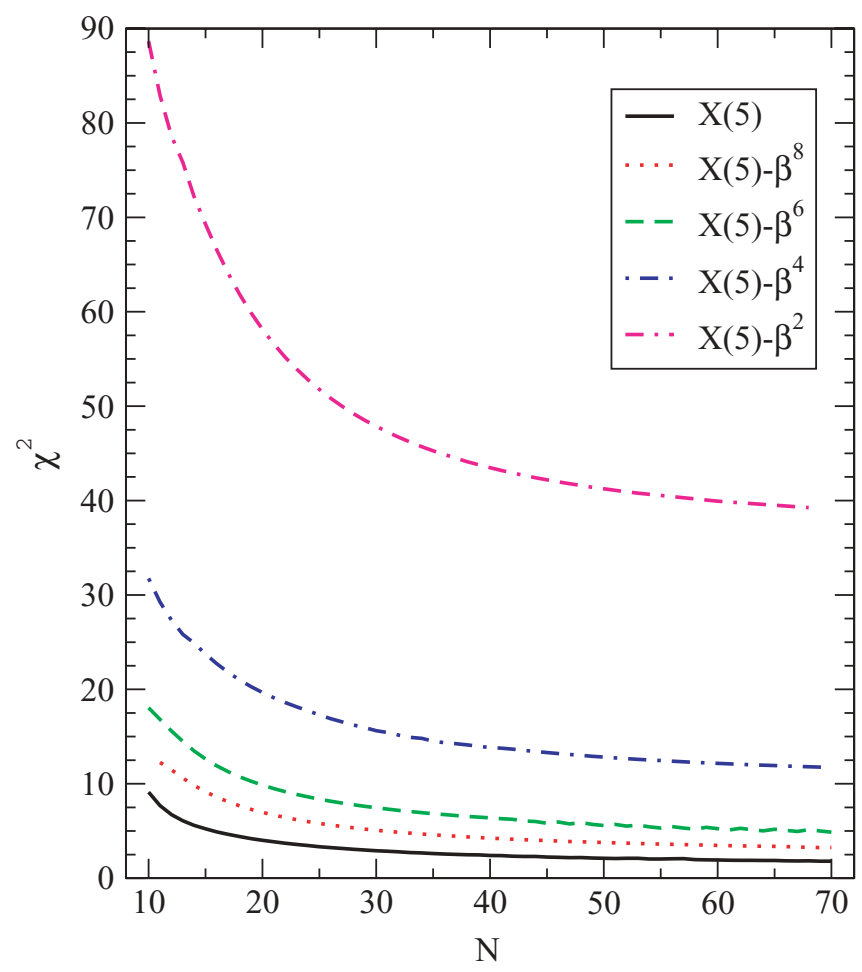

FIG. 7. (Color online) $\chi^{2}$ values for the $E 2$ transition rates for the different $\mathrm{X}(5)$ models, as a function of $N$, calculated using the IBM electromagnetic transition operator $\hat{T}_{M}^{E 2}=e_{\text {eff }}\left[\left(s^{\dagger} \tilde{d}+d^{\dagger} \tilde{s}\right)-\right.$ $\left.\sqrt{7} / 2\left(d^{\dagger} \times \tilde{d}\right)_{M}^{(2)}\right]$.

and thus no free parameters are left in this calculation. For the $B(E 2)$ values calculated (not a fit) a $\chi^{2}$ value has been obtained for each $\mathrm{X}(5)$ model with an arbitrary $\sigma=10$. In Fig. 7 the corresponding $\chi^{2}$ value is plotted as a function of $N$ for all the $\mathrm{X}(5)$ models considered. Figure 7 shows a smooth dependence of $\chi^{2}$ on $N$. The $\chi^{2}$ value decreases monotonically as $N$ increases for all the $\mathrm{X}(5)$ models. The best agreement is obtained for $\mathrm{X}(5)$ while the worst is for $\mathrm{X}(5)-\beta^{2}$.

For a quantitative comparison, the $B(E 2)$ values for selected transitions with $N=50$ are shown in Table IV. In this table, the remarkable agreement between the IBM calculations

TABLE IV. $B(E 2)$ values (in arbitrary units) obtained, for $N=50$, for the fitted IBM Hamiltonians (see text) compared with those provided by the different X(5) models.

\begin{tabular}{|c|c|c|c|c|c|c|c|c|c|c|}
\hline & $X(5)$ & IBM & $\mathrm{X}(5)-\beta^{8}$ & IBM & $\mathrm{X}(5)-\beta^{6}$ & IBM & $X(5)-\beta^{4}$ & IBM & $\mathrm{X}(5)-\beta^{2}$ & IBM \\
\hline$B\left(E 2: 2_{1}^{+} \rightarrow 0_{1}^{+}\right)$ & 100.0 & 100.0 & 100.0 & 100.0 & 100.0 & 100.0 & 100.0 & 100.0 & 100.0 & 100.0 \\
\hline$B\left(E 2: 4_{1}^{+} \rightarrow 2_{1}^{+}\right)$ & 159.9 & 155.4 & 163.4 & 159.1 & 165.3 & 145.7 & 169.0 & 164.9 & 177.9 & 174.6 \\
\hline$B\left(E 2: 6_{1}^{+} \rightarrow 4_{1}^{+}\right)$ & 198.2 & 181.6 & 208.8 & 189.2 & 214.6 & 160.8 & 226.2 & 199.9 & 255.2 & 217.7 \\
\hline$B\left(E 2: 8_{1}^{+} \rightarrow 6_{1}^{+}\right)$ & 227.6 & 199.1 & 247.3 & 210.3 & 258.1 & 166.2 & 279.9 & 224.9 & 337.1 & 249.2 \\
\hline$B\left(E 2: 0_{2}^{+} \rightarrow 2_{1}^{+}\right)$ & 62.4 & 43.4 & 74.7 & 55.7 & 81.0 & 11.2 & 93.2 & 74.6 & 121.9 & 106.7 \\
\hline$B\left(E 2: 2_{2}^{+} \rightarrow 2_{1}^{+}\right)$ & 8.2 & 4.4 & 9.7 & 7.3 & 10.3 & 0.9 & 11.3 & 15.4 & 13.4 & 33.4 \\
\hline$B\left(E 2: 2_{2}^{+} \rightarrow 0_{1}^{+}\right)$ & 2.1 & 0.2 & 2.2 & 0.0 & 2.2 & 3.5 & 2.0 & 0.6 & 1.6 & 4.4 \\
\hline$B\left(E 2: 2_{2}^{+} \rightarrow 0_{2}^{+}\right)$ & 79.5 & 56.1 & 97.2 & 67.5 & 106.0 & 68.3 & 122.0 & 69.3 & 155.7 & 49.2 \\
\hline$B\left(E 2: 4_{2}^{+} \rightarrow 2_{1}^{+}\right)$ & 0.9 & 0.2 & 0.8 & 0.0 & 0.7 & 5.2 & 0.5 & 0.7 & 0.1 & 2.1 \\
\hline$B\left(E 2: 4_{2}^{+} \rightarrow 4_{1}^{+}\right)$ & 6.1 & 3.4 & 7.7 & 6.8 & 8.4 & 0.7 & 9.6 & 16.6 & 12.4 & 27.7 \\
\hline$B\left(E 2: 4_{2}^{+} \rightarrow 2_{2}^{+}\right)$ & 120.0 & 111.9 & 149.1 & 121.7 & 162.9 & 108.1 & 187.7 & 110.9 & 240.3 & 99.3 \\
\hline
\end{tabular}


and those from the $\mathrm{X}(5)$ model become clear. However, as soon as we move to the rest of models the agreement starts getting worse. Some comments on these results are in order. First, one observes that the $B(E 2)$ values within the ground-state band are calculated qualitatively correct in all models. This agreement is quantitatively good for the $\mathrm{X}(5)$ case and gets worse for the other models. The $\mathrm{X}(5)-\beta^{2}$ case is the worst, showing differences of a factor of 2 for some transitions. The IBM seems to saturate to too small a value as the angular momentum increases; therefore the deviation increases with the angular momentum. The intraband transitions in the $\beta$ band exhibit the same kind of agreement, where the larger discrepancy is for $B\left(E 2: 4_{2}^{+} \rightarrow 2_{2}^{+}\right)$. The interband transitions once more agree well qualitatively. In summary, one can say that the structure of the wave functions is correctly captured by the IBM fit, especially for X(5), and the calculations are able to reproduce the sequence of large and small values (with few exceptions), confirming the appropriate structure of the wave functions.

\section{THE CRITICAL HAMILTONIAN}

One of the most attractive features of the $\mathrm{X}(5)$ models treated in this work is that they are supposed to describe, at different approximation levels, the critical point in the transition from spherical to rigid axially deformed shapes. Since they are connected to a given IBM Hamiltonian, as shown in the preceding section, this should correspond to the critical point in the transition from spherical to axially deformed shapes, that is, this Hamiltonian should produce an energy surface with degenerated spherical and deformed minima. Is this the case for the fitted IBM Hamiltonians obtained in the preceding section? Before starting with the discussion it is necessary to establish a measure of how close is a given IBM Hamiltonian to the critical point.

An energy surface can be associated with a given IBM Hamiltonian by using the intrinsic state formalism [8,9,11], which introduces the shape variables $(\beta, \gamma)$ in the IBM. To define the intrinsic state one has to consider that the dynamical behavior of the system can be approximately described in terms of independent bosons moving in an average field [50]. The ground state of the system is written as a condensate, $|c\rangle$, of bosons that occupy the lowest energy phonon state, $\Gamma_{c}^{\dagger}$ :

$$
|c\rangle=\frac{1}{\sqrt{N !}}\left(\Gamma_{c}^{\dagger}\right)^{N}|0\rangle
$$

where

$\Gamma_{c}^{\dagger}=\frac{1}{\sqrt{1+\beta^{2}}}\left(s^{\dagger}+\beta \cos \gamma d_{0}^{\dagger}+\frac{1}{\sqrt{2}} \beta \sin \gamma\left(d_{2}^{\dagger}+d_{-2}^{\dagger}\right)\right)$.

Here $\beta$ and $\gamma$ are variational parameters related with the shape variables in the geometrical collective model [11]. The expectation value of the Hamiltonian (1) in the intrinsic state (10) provides the energy surface of the system, $E(N, \beta, \gamma)=\langle c|\hat{H}| c\rangle$. This energy surface in terms of the parameters of the Hamiltonian (1) and the shape variables can be readily obtained [51] (where we keep the $\kappa_{4}$ variable for completeness) as

$$
\begin{aligned}
\langle c|\hat{H}| c\rangle= & \frac{N \beta^{2}}{\left(1+\beta^{2}\right)}\left(\varepsilon_{d}+6 \kappa_{1}-\frac{9}{4} \kappa_{2}+\frac{7}{5} \kappa_{3}+\frac{9}{5} \kappa_{4}\right) \\
& +\frac{N(N-1)}{\left(1+\beta^{2}\right)^{2}}\left[\frac{\kappa_{0}}{4}+\beta^{2}\left(-\frac{\kappa_{0}}{2}+4 \kappa_{2}\right)\right. \\
& \left.+2 \sqrt{2} \beta^{3} \kappa_{2} \cos (3 \gamma)+\beta^{4}\left(\frac{\kappa_{0}}{4}+\frac{\kappa_{2}}{2}+\frac{18}{35} \kappa_{4}\right)\right] .
\end{aligned}
$$

The shape of the nucleus is defined through the equilibrium value of the deformation parameters, $\beta$ and $\gamma$, which are obtained by minimizing the ground-state energy, $\langle c|\hat{H}| c\rangle$. A spherical nucleus has a global minimum on the energy surface at $\beta=0$, whereas a deformed one has the absolute minimum at a finite value of $\beta$. The parameter $\gamma$ represents the departure from axial symmetry; that is, $\gamma=0$ and $\gamma=\pi / 3$ stand for a prolate and oblate, respectively, axially deformed nucleus, while any other value corresponds to a triaxial shape. An additional situation arises when the energy surface is independent of $\gamma$ but shows a minimum at a finite value of $\beta$; in this case the nucleus is $\gamma$-unstable. It has to be noted that for a general IBM Hamiltonian including up to two-body terms, such as the one considered in this work, triaxiality is forbidden.

With the tools just described one can study phase transitions in the IBM [8]. First, the parameters that define the Hamiltonian are the control parameters and these are usually chosen in such a way that only one of them is a variable, while the rest remain constant. The deformation parameters $\beta$ and $\gamma$ become the order parameters, although in our case the order parameter is just $\beta$. Roughly speaking, a phase transition appears when there exists an abrupt change in the shape of the system when changing smoothly the control parameter. The phase transitions can be classified according to the Ehrenfest classification [52]. First-order phase transitions appear when there exists a discontinuity in the first derivative of the energy with respect to the control parameter. This discontinuity appears when two degenerate minima exist in the energy surface for two values of the order parameter $\beta$. Second-order phase transitions appear when the second derivative of the energy with respect to the control parameter displays a discontinuity. This happens when the energy surface presents a single minimum for $\beta=0$ and the surface satisfies the condition $\left(\frac{d^{2} E}{d \beta^{2}}\right)_{\beta=0}=0$. In a modern classification, secondorder phase transitions belongs to the high-order or continuous phase transitions [52]. The $\mathrm{X}(5)$ situation was designed to describe first-order phase transitions.

To determine whether a given Hamiltonian corresponds to a critical point or not, the flatness or the existence of two degenerate minima in the energy surface should be investigated. For the case of a one-parameter IBM Hamiltonian (e.g., consistent Q (CQF) Hamiltonians [53]), it is simple to find an analytical expression for the critical control parameter in the Hamiltonian. However, for a general IBM Hamiltonian, such as the one used in this work, it is necessary to rewrite the energy surface in a special way, as proposed first by López-Moreno and Castaños [15]. There, the authors manage 
to write the energy surface of a general IBM Hamiltonian in terms of two parameters. They made use of some concepts from catastrophe theory [54] to define the two essential parameters, $\left(r_{1}, r_{2}\right)$ of the problem. In terms of these two essential parameters they found expressions for the locus, in the essential parameter space, that gives a critical point at the origin in $\beta$, called the bifurcation set, and for the locus that gives rise to two degenerate minima, called the Maxwell set. The essential parameters $r_{1}$ and $r_{2}$ can be written as

$$
\begin{aligned}
& r_{1}=\frac{a_{3}-u_{0}+\tilde{\varepsilon} /(N-1)}{2 a_{1}+\tilde{\varepsilon} /(N-1)-a_{3}}, \\
& r_{2}=-\frac{2 a_{2}}{2 a_{1}+\tilde{\varepsilon} /(N-1)-a_{3}},
\end{aligned}
$$

where

$$
\begin{aligned}
\tilde{\varepsilon} & =\varepsilon_{d}+6 \kappa_{1}-\frac{9}{4} \kappa_{2}+\frac{7}{5} \kappa_{3}+\frac{9}{5} \kappa_{4}, \\
a_{1} & =\frac{1}{4} \kappa_{0}+\frac{1}{2} \kappa_{2}+\frac{18}{35} \kappa_{4}, \\
a_{2} & =2 \sqrt{2} \kappa_{2}, \\
a_{3} & =-\frac{1}{2} \kappa_{0}+4 \kappa_{2}, \\
u_{0} & =\frac{\kappa_{0}}{2} .
\end{aligned}
$$

By using the essential parameters, the energy surface can be written as

$$
\begin{aligned}
E^{*}(\beta, \gamma) & =\frac{\langle c|\hat{H}| c\rangle-N(N-1) u_{0} / 2}{N(N-1)\left(2 a_{1}+\tilde{\varepsilon} /(N-1)-a_{3}\right)} \\
& =\frac{1}{1+\beta^{2}}\left[\beta^{4}+r_{1} \beta^{2}\left(\beta^{2}+2\right)-r_{2} \beta^{3} \cos 3 \gamma\right] .
\end{aligned}
$$

Note that $E^{*}(\beta, \gamma)$ does not depend on the number of bosons, allowing us to compare fairly energy surfaces corresponding to different boson numbers.

In Fig. 8 we display the plane of the essential parameters where the fitted Hamiltonians to the different $X(5)$ models are plotted with different symbols. In this plane a critical first-order Hamiltonian corresponds to a point over the dashed line (Maxwell set, i.e., two degenerated minima). The $r_{1}=0$ line corresponds to $\left(\frac{d^{2} E}{d \beta^{2}}\right)_{\beta=0}=0$ (i.e., to the appearance of the spherical minimum called antispinodal point). The curved full line above the Maxwell set corresponds to $\left(\frac{d^{2} E}{d \beta^{2}}\right)_{\beta_{0}}=0$, with $\beta_{0} \neq 0$ (i.e., it corresponds to the appearance of a deformed minimum called spinodal point). The area below the curved full line and above $r_{1}=0$ is the coexistence region. In this region two minima, one spherical and one deformed, coexist. We also represent in the plane the second-order critical point $\left(r_{1}=r_{2}=0\right)$. Note that all the points above the dashed line correspond to spherical shapes, whereas those below that line represent deformed shapes. We only plot the $r_{2}>0$ semiplane because our IBM Hamiltonian always corresponds to prolate shapes and therefore to $r_{2}>0$. The semiplane $r_{2}<0$ is identical to the one presented in Fig. 8, but for oblate shapes. The different symbols in the figure correspond to the IBM Hamiltonians fitted to one of the $\mathrm{X}(5)$ models (see legend) for $N$ ranging from 10 to 70 . The idea is to see whether

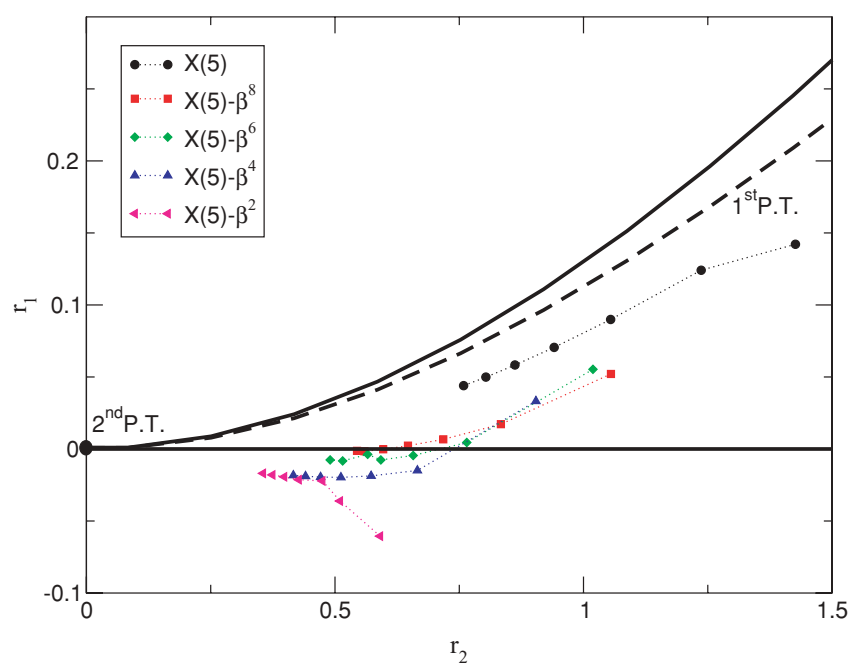

FIG. 8. (Color online) Position in the plane $r_{1}-r_{2}$ of the energy surfaces extracted from the fitted IBM Hamiltonians to the different $\mathrm{X}(5)$ models as a function of $N(10,20,30,40,50,60$, and 70). The largest value of $N$ is placed on the left-hand side whereas the smallest one is on the right part of the figure.

in the large- $N$ limit the obtained IBM Hamiltonians go to the Maxwell set (dashed line), which is the line of first-order phase transitions. For all the models, the $N$ value of the different points increases from the right to the left.

Several important features can be extracted from this figure. The main one is that the $\mathrm{X}(5)$ case, for the whole range of $N$, is always very close to the Maxwell set (i.e., to the first-order phase transition line), being closer as the value of $N$ increases. This is not exactly the case for the rest of fits. $\mathrm{X}(5)-\beta^{8}, \mathrm{X}(5)-\beta^{6}$, and $\mathrm{X}(5)-\beta^{4}$ are situated on the coexistence region for small or moderate $N$ values, but they go toward the prolate deformation region as $N$ increases, although they are always very close to the antispinodal line. Finally, the case $\mathrm{X}(5)-\beta^{2}$ lies in the deformed region but it approaches the coexistence area as $N$ increases. In general, one observes that the mapped IBM energy surfaces move further away from the coexistence region as one changes from $\mathrm{X}(5)$ through $\mathrm{X}(5)-\beta^{8}, \mathrm{X}(5)-\beta^{6}, \mathrm{X}(5)-\beta^{4}$, and $\mathrm{X}(5)-\beta^{2}$. In general, the higher the value of $n$ is $\left[\mathrm{X}(5)-\beta^{n}\right]$, the closer to the coexistence region is the IBM energy surface. In all the cases one observes that the different models always move in the direction of the second-order phase transition point as $N$ increases.

To illustrate graphically the shapes of the energy surfaces obtained with the different IBM Hamiltonians, we plot in Fig. 9 the axial IBM energy surfaces $E^{*}(\beta, 0)$ [Eq. (16)], along the prolate leg, extracted from the fit to the five analyzed $\mathrm{X}(5)$ models as a function of the deformation $\beta$ for three values of $N: 10,40$, and 70 . All the panels show a rather similar aspect: For $N=10$ there exists a more pronounced minimum, while passing from $N=40$ to $N=70$ the energy surface flattens, moving into a shape with two approximately degenerated minima. As was studied in Fig. 8, in Fig. 9(a), which is for $X(5)$, the three curves present two minima, in Figs. 9(b), 9(c), and $9(\mathrm{~d})$, corresponding to $\mathrm{X}(5)-\beta^{8}, \mathrm{X}(5)-\beta^{6}$, and $\mathrm{X}(5)-\beta^{4}$, respectively, only the line for $N=10$ has two minima, whereas 


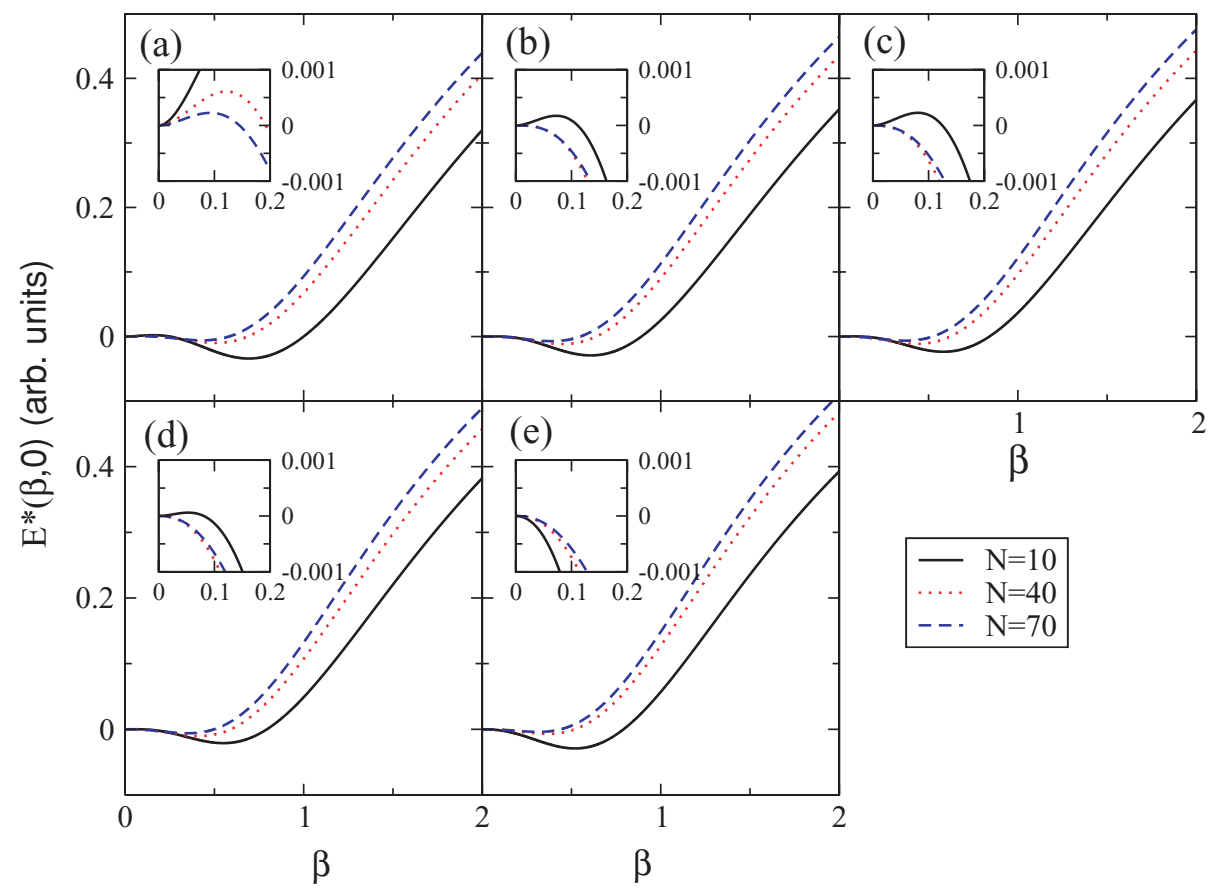

FIG. 9. (Color online) IBM axial energy surfaces, for prolate shapes [see Eq. (16)] as a function of $\beta$, for selected values of $N$ (see text for definition). (a) $\mathrm{X}(5)$, (b) $\mathrm{X}(5)-\beta^{8}$, (c) $\mathrm{X}(5)-\beta^{6}$, (d) $\mathrm{X}(5)-\beta^{4}$, and (e) $\mathrm{X}(5)-\beta^{2}$. The insets show closer views of the region around $\beta=0$.
$N=40$ and $N=70$ show only a deformed minimum (see insets of Fig. 9). Finally, Fig. 9(e) is for $\mathrm{X}(5)-\beta^{2}$, and the three curves present only a deformed minimum, although it clearly flattens as $N$ increases. This analysis confirms that $\mathrm{X}(5)$ models map into IBM Hamiltonians that are very close to the first-order phase transition line, but the closest Hamiltonian to the transition area is the one mapped from the original $\mathrm{X}(5)$ model.

A somewhat similar analysis to the one presented in this section was performed in $[42,43]$. There, the authors studied the connection between $\mathrm{X}(5)$ and $\mathrm{X}(5)-\beta^{2}$ using a two-parameter IBM Hamiltonian [and they also analyzed $\mathrm{X}(3)]$ for $N=10$. Their conclusion was that the $\mathrm{X}(5)$ case is close to, although not exactly at, the first-order phase transition region for a finite number of bosons. This conclusion is very similar to the one extracted from our results. For the case of $\mathrm{X}(5)-\beta^{2}$ their results are similar to the $\mathrm{X}(5)$ case; that is, the corresponding IBM Hamiltonian is even closer to the first-order phase transition line that in the $\mathrm{X}(5)$ case. These latter results are also in agreement with the conclusions raised in the present work, although we have found that $\mathrm{X}(5)-\beta^{2}$ is further from the coexistence region than $X(5)$. The origin of the discrepancy should be based on the more restricted set of data used in $[42,43]$ in their fits and the smaller number of parameters used in the IBM Hamiltonian.

\section{SUMMARY AND CONCLUSIONS}

In this paper, we have studied the connection between the $\mathrm{X}(5)$ models and the IBM on the basis of a numerical mapping between these models. To establish the mapping we have performed a best fit of the general IBM Hamiltonian to a selected set of energy levels produced by several $\mathrm{X}(5)$ models; as an additional parameter we have used the energy of the $\gamma$ bandhead, which is not fixed by the $\mathrm{X}(5)$ models. Later, a free parameter check of the wave functions, obtained with the best-fit parameters, was done by calculating relevant $B(E 2)$ transition rates. All calculations have been done as a function of the number of bosons. Once the best-fit IBM Hamiltonians to the different $\mathrm{X}(5)$ models are obtained, their energy surfaces are constructed and analyzed with the help of catastrophe theory so as to learn how close they are to a critical point.

We have shown that it is possible, in all cases, to obtain a mapping between the $\mathrm{X}(5)$ models and the IBM with a reasonable agreement for both energies and $B(E 2)$ transition rates. In general, the goodness of the fit to the energies and $B(E 2)$ transition rates is independent on the number of bosons. Globally, the agreement is similar for all the models: for the energies the best agreement is for $\mathrm{X}(5)-\beta^{8}$ and $\mathrm{X}(5)-\beta^{6}$ whereas the worst is for $\mathrm{X}(5)$. In any case, the $\chi^{2}$ values obtained that give the goodness of the fits are comparable for all the models. Additional tests have been done to the produced wave functions by calculating $B(E 2)$ transition rates. No free parameters are included in these calculations. In this case, the best agreement (smallest $\chi^{2}$ value) is obtained for $\mathrm{X}(5)$ whereas the worst is for $\mathrm{X}(5)-\beta^{2}$. A consequence of this good general agreement is that it would be almost impossible, from an experimental point of view, to discriminate between a $\mathrm{X}(5)$ model and its corresponding IBM Hamiltonian when only a few low-lying states are considered (usually the four lowest states in the ground, $\beta$, and $\gamma$ bands.)

We have also proved that the $\mathrm{X}(5)$ model corresponds to an IBM Hamiltonian that is very close to the first-order phase transition region, getting closer for larger values of $N$. X(5)- $\beta^{8}$, $\mathrm{X}(5)-\beta^{6}$, and $\mathrm{X}(5)-\beta^{4}$ models lie within the coexistence region for small and moderate values of $N$, but they move slightly into the deformed region for larger values of $N$. Finally, $\mathrm{X}(5)-\beta^{2}$ lies all the way into the deformed region, although very close 
to the coexistence region. In all the cases the system evolves toward the second-order critical point as $N$ increases.

It is worth mentioning that the conclusions raised in this work are somewhat dependent on the constraints imposed in the fitting procedure. On one hand we have checked that the inclusion, or not, of high-lying states in the bands considered in the fit does not strongly affect to the results. On the other hand we have extensively checked the case with $\kappa_{4} \neq 0$ [48]. This later case generates values of $\chi^{2}$ similar to the ones presented in this work, but the global picture of the mapped IBM Hamiltonians to the $\mathrm{X}(5)$ models is not so consistent as the one shown here. In particular, the change of the Hamiltonian parameters as a function of $N$ or as a function of the considered $\mathrm{X}(5)$ model is not smooth enough. There are instabilities in the fitting procedure because the produced $\chi^{2}$ surface is very flat.

Finally, it is worth mentioning the differences between the X(5) model-IBM and the E(5) model-IBM mappings [34]. For the $\mathrm{E}(5)$ models the agreement for both the energies and the $B(E 2)$ transition rates is really remarkable and much better than for the $\mathrm{X}(5)$ models. Globally, the best agreement is obtained for the E(5)- $\beta^{4}$ Hamiltonian and the worst for the $\mathrm{E}(5)$ case. For the case of very large number of bosons the only E(5) model that can be reproduced exactly by the IBM is $\mathrm{E}(5)-\beta^{4}$, corresponding such an IBM Hamiltonian with the critical point of the model $\left(r_{1}=0\right)$ (as shown in Refs. [55,56]). All the $\mathrm{E}(5)$ models correspond to IBM Hamiltonians very close to the critical area, $\left|r_{1}\right|<0.05$ with $r_{2}=0$. Therefore, one can say that the $\mathrm{E}(5)$ models are appropriate to describe transitional $\gamma$-unstable regions close to the critical point. However, not all the $\mathrm{X}(5)$ models are suitable for describing the critical area between the axially deformed and the spherical shapes; only $\mathrm{X}(5)$ is really appropriate to this end. Finally, for the E(5) models the existence of something similar to a quasidynamical symmetry [57] is observed, a phenomenon we call quasi-critical point symmetry. In the case of the X(5) models we cannot talk about quasi-critical point symmetry because the agreement between the IBM and the X(5) models is not good enough; only in the case of the ground-state band for X(5) do we have the appropriate agreement to say that a quasi-critical point symmetry is present.

\section{ACKNOWLEDGMENTS}

This work has been partially supported by the Spanish Ministerio de Educación y Ciencia and by the European Regional Development Fund (FEDER) under Project Nos. FIS2008-04189 and FPA2007-63074, by CPAN-Ingenio, and by the Junta de Andalucía under Project Nos. FQM160, FQM318, P05-FQM437, and P07-FQM-02962.
[1] A. Bohr, Mat. Fys. Medd. K. Dan. Vidensk. Selsk. 26, 14 (1952).

[2] A. Bohr and B. R. Mottelson, Mat. Fys. Medd. K. Dan. Vidensk. Selsk. 27, 16 (1953).

[3] A. Bohr and B. R. Mottelson, Nuclear Structure, Vol. II (Benjamin, Elmsford, NY, 1969).

[4] A. Arima and F. Iachello, Ann. Phys. (NY) 99, 253 (1976).

[5] A. Arima and F. Iachello, Ann. Phys. (NY) 111, 201 (1978).

[6] O. Scholten, F. Iachello, and A. Arima, Ann. Phys. (NY) 115, 325 (1978).

[7] F. Iachello and A. Arima, The Interacting Boson Model (Cambridge University Press, Cambridge, UK, 1987).

[8] A. E. L. Dieperink, O. Scholten, and F. Iachello, Phys. Rev. Lett. 44, 1747 (1980).

[9] A. E. L. Dieperink and O. Scholten, Nucl. Phys. A 346, 125 (1980).

[10] J. N. Ginocchio and M. W. Kirson, Phys. Rev. Lett. 44, 1744 (1980).

[11] J. N. Ginocchio and M. W. Kirson, Nucl. Phys. A 350, 31 (1980).

[12] M. W. Kirson, Ann. Phys. (NY) 143, 448 (1982).

[13] J. N. Ginocchio, Nucl. Phys. A 376, 438 (1982).

[14] D. J. Rowe and G. Thiamova, Nucl. Phys. A 760, 59 (2005).

[15] E. López-Moreno and O. Castaños, Phys. Rev. C 54, 2374 (1996).

[16] J. M. Arias, J. Dukelsky, J. E. García-Ramos, and J. Vidal, Phys. Rev. C 75, 014301 (2007).

[17] D. J. Rowe, P. S. Turner, and G. Rosensteel, Phys. Rev. Lett. 93, 232502 (2004).

[18] D. J. Rowe, Nucl. Phys. A 745, 47 (2004).

[19] P. S. Turner and D. J. Rowe, Nucl. Phys. A 756, 333 (2004).

[20] G. Thiamova and D. J. Rowe, Eur. Phys. J. A 41, 189 (2009).

[21] D. L. Hill and J. A. Wheeler, Phys. Rev. 89, 1102 (1953).
[22] J. M. Eisenberg and G. Greiner, Nuclear Models (NorthHolland/American Elsevier, Amsterdam/New York, 1970).

[23] D. Janssen, R. V. Jolos, and F. Dönau, Nucl. Phys. A 224, 93 (1974)

[24] M. Moshinsky, Nucl. Phys. A 338, 156 (1980).

[25] A. Klein and M. Vallieres, Phys. Lett. B 98, 5 (1981).

[26] A. Klein, C.-T. Li, and M. Vallieres, Phys. Rev. C 25, 2733 (1982).

[27] H. J. Assenbaum and A. Weiguny, Z. Phys. A 310, 75 (1983).

[28] P. Van Isacker, Phys. Rev. Lett. 83, 4269 (1999).

[29] F. Iachello, Phys. Rev. Lett. 85, 3580 (2000).

[30] F. Iachello, Phys. Rev. Lett. 87, 052502 (2001).

[31] F. Iachello, Phys. Rev. Lett. 91, 132502 (2003).

[32] R. F. Casten and N. V. Zamfir, Phys. Rev. Lett. 85, 3584 (2000).

[33] R. F. Casten and N. V. Zamfir, Phys. Rev. Lett. 87, 052503 (2001).

[34] J. E. García-Ramos and J. M. Arias, Phys. Rev. C 77, 054307 (2008).

[35] R. F. Casten and E. A. McCutchan, J. Phys. G 34, R285 (2007), and references therein.

[36] P. Cejnar, J. Jolie, and R. F. Casten, Rev. Mod. Phys. 82, 2155 (2010).

[37] P. G. Bizzeti and A. M. Bizzeti-Sona, Phys. Rev. C 70, 064319 (2004).

[38] P. G. Bizzeti and A. M. Bizzeti-Sona, Phys. Rev. C 77, 024320 (2008).

[39] D. Bonatsos, D. Lenis, N. Minkov, D. Petrellis, and P. Yotov, Phys. Rev. C 71, 064309 (2005).

[40] D. Bonatsos, D. Lenis, N. Minkov, P. P. Raychev, and P. A. Terziev, Phys. Rev. C 69, 014302 (2004).

[41] D. Bonatsos, D. Lenis, D. Petrellis, P. A. Terziev, and I. Yigitoglu, Phys. Lett. B 632, 238 (2006). 
[42] E. A. McCutchan, N. V. Zamfir, and R. F. Casten, Phys. Rev. C 71, 034309 (2005).

[43] E. A. McCutchan, D. Bonatsos, and N. V. Zamfir, Phys. Rev. C 74, 034306 (2006).

[44] D. Bonatsos et al., Phys. Rev. C 76, 064312 (2007).

[45] D. Bonatsos, D. Lenis, E. A. McCutchan, D. Petrellis, and I. Yigitoglu, Phys. Lett. B 649, 394 (2007).

[46] M. A. Caprio, Phys. Rev. C 72, 054323 (2005).

[47] A. Frank and P. Van Isacker, Algebraic Methods in Molecular and Nuclear Structure Physics (Wiley, New York, 1994).

[48] J. Barea et al. (unpublished).

[49] F. James, MINUIT: Function Minimization and Error Analysis Reference Manual, Version 94.1, CERN, 1994.
[50] J. Dukelsky, G. G. Dussel, R. P. J. Perazzo, S. L. Reich, and H. M. Sofia, Nucl. Phys. A 425, 93 (1984).

[51] P. Van Isacker and J. Q. Chen, Phys. Rev. C 24, 684 (1981).

[52] H. E. Standley, Introduction to Phase Transitions and Critical Phenomena (Oxford University Press, Oxford, 1971).

[53] D. D. Warner and R. F. Casten, Phys. Rev. Lett. 48, 1385 (1982).

[54] R. Gilmore, Catastrophe Theory for Scientists and Engineers (Wiley, New York, 1981).

[55] J. M. Arias, C. E. Alonso, A. Vitturi, J. E. García-Ramos, J. Dukelsky, and A. Frank, Phys. Rev. C 68, 041302(R) (2003).

[56] J. E. García-Ramos, J. Dukelsky, and J. M. Arias, Phys. Rev. C 72, 037301 (2005).

[57] D. J. Rowe, Phys. Rev. Lett. 93, 122502 (2004). 